\title{
El Contraejemplo en la Elaboración de la Definición de Función Convexa por Estudiantes Universitarios
}

\author{
Juan C. Hernández-Gómez ${ }^{(1) *}$, Edgardo Locia-Espinoza( ${ }^{(1)}$, Armando Morales-Carballo ${ }^{(1)} y$ \\ José M.Sigarreta-Almira(1) \\ (1) Universidad Autónoma de Guerrero, Avenida Lázaro Cárdenas S/N, Ciudad Universitaria, Chilpancingo, \\ Gro. México. (e-mail: jcarloshg@gmail.com, lociae999@hotmail.com, armando280@hotmail.com, \\ josemariasigarretaalmira@hotmail.com)
}

${ }^{*}$ Autor a quien debe ser dirigida la correspondencia

Recibido Abr. 24, 2018; Aceptado Jun. 25, 2018; Versión final Ago. 29, 2018, Publicado Feb. 2019

\begin{abstract}
Resumen
En este artículo se reportan los resultados de una investigación que tuvo como objetivo identificar el papel que juega el contraejemplo en la elaboración de definiciones matemáticas. En particular se analizó el caso de la función convexa en un grupo de estudiantes de nivel universitario. Los elementos teóricos y metodológicos primarios que sustentaron el trabajo descansan en los aportes sobre pruebas y refutaciones, elementos de heurística, lenguaje y axiomática y en el debate científico en cursos de matemáticas. Como resultado fundamental se destaca que el debate científico favoreció mediante la discusión en torno al concepto de función convexa y a la formulación de contraejemplos. Estos contribuyeron en la comprobación de casos particulares, la formulación y refutación de conjeturas, hasta aproximarse a la presentación clásica de la definición. Establecemos también que el contraejemplo es un recurso didáctico y una herramienta mediadora que contribuye en los procesos de elaboración y comprensión de la definición matemática.
\end{abstract}

Palabras clave: contraejemplo; debate científico; función convexa; definición generada por prueba.

\section{The Counterexample in the Elaboration of the Definition of the Convex Function for University Students}

\begin{abstract}
In this paper the results of an investigation that aimed to identify the role played by the counterexample in the elaboration of mathematical definitions are reported. In particular, the case of the convex function; in a group of university students was analyzed. The principal theoretical and methodological elements that supported the work rest on the contributions in proof and refutations, elements of heuristics, language and axiomatics and in the scientific debate in mathematics courses. As a fundamental result, we emphasized that the scientific debate favored, through the discussion on the concept of convex function and the formulation of counterexamples These contributed to the verification of particular cases, the formulation and refutation of conjectures, until approaching the classic presentation of the definition. We also establish that the counterexample is a didactic resource and a mediation tool that contributes in the processes of elaboration and understanding of the mathematical definition.
\end{abstract}

Keywords:counterexample; scientific debate; convex function; definition generated by proof 


\section{INTRODUCCIÓN}

Varios autores coinciden en la importancia de las conjeturas y sus refutaciones vistas a través del contraejemplo como una vía para la reconstrucción del conocimiento y desarrollo del pensamiento matemático. Además, consideran que el contraejemplo puede ser una herramienta mediadora que posibilite una alternativa para favorecer los procesos de enseñanza y aprendizaje de contenidos de la matemática que son tratados en los distintos niveles educativos (Komatsu, 2010; García y Morales, 2013; Martínez y Detzel, 2010; Bustos y Zubieta, 2015). En las situaciones prácticas de los procesos de enseñanza y aprendizaje, el contraejemplo actúa como una fuerza de impulso que obliga a refinar las conjeturas o a refutarlas, este proceso favorece el aspecto formal en la presentación del contenido matemático.

En la presente investigación asumimos como definición de contraejemplo la dada por S.Kleene: "Una fórmula $F$ del cálculo de predicados no es válida exactamente si $F$ es falsable en el sentido siguiente: existe un dominio no vacío $D$ y una asignación de los parámetros de $F$ que da el valor f(falso). Tal asignación será llamada asignación falsificante para $F$ en $D$, y F será llamada falsable en este $D$. El sistema formado por este $D$ y esta asignación podrá ser designado como un contraejemplo de F”. (Kleene, 1967, p.284). En la definición dada, consideramos al contraejemplo como una par $(D, \square)$ formada por un dominio $D$ y una asignación $\square \square$ (es decir una n-tupla de valores de los parámetros de F), para que esta pareja, sea un ejemplo o un contraejemplo, es necesario remitirla al argumento F: por consecuencia ( $D \square \square \square \mathrm{F}$ ) forman un contraejemplo si $\square \square \in \mathrm{D}$ y si $\square \square \mathrm{F}$ ) toma el valor $\mathrm{f}$. La formulación natural en matemáticas se transforma en " $\square \square$ es el contraejemplo de $\mathrm{F}$ en el dominio D". En este caso el uso pone el acento sobre la asignación $\square$, que se transforma en "el contraejemplo", mientras que los otros dos términos son relegados al segundo plano como condiciones o incluso se funden en el contexto.

En el campo de la enseñanza-aprendizaje de la matemática se han desarrollado trabajos relativos a la formulación de conjeturas y al empleo del contraejemplo para el tratamiento de conceptos, propiedades y relaciones matemáticas. En particular, en las investigaciones reportadas por Weber (2009), Komatsu (2010), Knuth y Ko(2013), Giannakoulias, Mastorides, Potari y Zachariades, (2010), Komatsu, Jones, Ikeda y Narazaki (2017),García y Morales (2013), Klymchuk, (2010), Zazkis y Chernoff (2008), Huang (2014) se han identificado que las mismas van dirigidas a profesores y estudiantes, en cada una de ellas se pone de manifiesto que la elaboración y utilización del contraejemplo es una herramienta didáctica para favorecer los procesos de validación y comprensión del conocimiento matemático.

Del análisis de los trabajos, anteriormente citados, respecto al uso del contraejemplo; identificamos las siguientes implicaciones metodológicas: 1) Permite estimular el razonamiento en los estudiantes del cómo y el porqué de los procesos que se siguen para llegar a conclusiones, y disminuir los procedimientos memorísticos y algorítmicos de aprendizaje: esta implicación se identificó en los trabajos de investigación dirigidos hacia el alumno en los niveles escolares (primaria, secundaria, bachillerato, licenciatura y posgrado) al tratar contenidos específicos de la matemática tales como: aritmética, álgebra, geometría, cálculo y análisis, teoría de números, entre otras; 2) Permite la estructuración funcional de los razonamientos lógicomatemáticos: esta implicación fue identificada en las propuestas relacionadas con el estudio de la prueba y la producción del contraejemplo para la validación de resultados; 3) Favorece la reflexión de los estudiantes en aspectos esenciales de las matemáticas, al poner de manifiesto la importancia de las reglas, principio, teoremas y propiedades asociadas a los objetos matemáticos: esta implicación fue identificada en la actividad del alumno, del profesor y del profesor en formación; 4) Permite identificar a través del proceso constructivo del conocimiento las características y propiedades básicas e invariantes de los objetos matemáticos: esta implicación se identificó a partir de propuestas de contraejemplos que favorecieron la refutación de conjeturas sobre definición de conceptos de objetos matemáticos en temas concretos de aritmética, geometría, álgebra, cálculo, teoría de números, entre otras; 5) Revela conceptos erróneos, y obliga a prestar atención a cada detalle del proceso mejorando la comprensión de los conceptos y propiedades matemáticas: esta implicación se identificóal concebir el contraejemplo en su connotación semántica y su uso como herramienta didáctica para favorecer los procesos de validación; y en dicho proceso, la refutación mediante la propuesta de contraejemplo fue fundamental.

En el presente trabajo se reconocen los aportes de (Polya, 1958) y (Lakatos, 1984) en los que establecen que el contraejemplo ha jugado un papel central a lo largo de la historia del desarrollo del conocimiento matemático, pues su inserción permitió la refinación, y/o refutación de conjeturas sobre conceptos y sus definiciones, propiedades y sus relaciones, hasta llegar a la presentación clásica de los contenidos que hoy se identifican en los libros de texto, esencialmente. En la misma dirección, se asume el papel metodológico del contraejemplo, en el contexto escolar, a partir de su relación funcional para el establecimiento de argumentos matemáticos, y de su imbricación didáctica, que constituye una actividad que los estudiantes necesitan practicar constantemente. Además, se pone de manifiesto los alcances metodológicos y relevancia del contraejemplo, y su utilidad en los procesos de elaboración del conocimiento matemático en el contexto 
escolar, por ello, enfatizan en su uso para favorecer en estudiantes de nivel licenciatura de la Universidad Autónoma de Guerrero los procesos de elaboración de la definición del concepto de función convexa, un contenido que se trabaja en los cursos de Cálculo, Análisis Matemático, y en áreas afines de aplicación de la Matemática, enmarcadas en el plan de estudios 2009.

Morales, Dolores, Hernández, Nolasco y Sigarreta (2014) y Ramos y López (2015) coinciden en establecer que los conceptos matemáticos son una categoría especial en la enseñanza de la Matemática, ya que constituyen la forma fundamental con que opera el pensamiento matemático. Con su formación se contribuye a la consecución del importante objetivo de las matemáticas: representar la relación entre la matemática y la realidad objetiva. En esta misma línea (Winicki, 2006) sostiene que los conceptos y sus definiciones, los axiomas y teoremas son la base de las estructuras de las distintas ramas de la matemática. Por tanto, en los procesos de enseñanza y aprendizaje se esperaría un tratamiento especial a los procesos de formación y definición de conceptos como actividades esenciales de la enseñanza y para el aprendizaje. Sin embargo, en la práctica no ocurre de este modo, generalmente en los procesos de enseñanza y aprendizaje de la matemática en los distintos niveles está ausente la actividad de reflexionar y orientar la actividad hacia la formación y definición de conceptos.

En los trabajos antes mencionados se han identificado cuatro razones principales, por las cuales es importante la elaboración de conceptos y definiciones en la enseñanza de la Matemática: 1) La comprensión de conceptos y definiciones matemáticos, es fundamental para el entendimiento de relaciones matemáticas; 2) Es una condición previa e importante para la capacidad de aplicar lo aprendido, en forma segura y creadora; 3) La formación de conceptos y definiciones claras, representa un punto esencial para el adiestramiento lógico-verbal; 4) La posibilidad de transmitir en la elaboración de conceptos, importantes nociones ideológicas y de la teoría del conocimiento, y de desarrollar una serie de valiosas cualidades de la personalidad.

Sustentados en lo anterior, identificamos la relevancia del tratamiento metodológico de los conceptos y sus definiciones como vía para el desarrollo del conocimiento matemático. Sin embargo, tal posición dista mucho de lo que sucede en las prácticas tradicionales de enseñanza-aprendizaje, ya que comúnmente se observa que en dichas prácticas hay repetición ante los alumnos de las definiciones tal cual vienen enunciadas en los libros de texto, sin duda que esta práctica afecta de manera negativa al aprendizaje de los alumnos con los cuales se abordan los determinados contenidos ya que no es suficiente para el entendimiento de los conceptos a que se refieren las definiciones.

A pesar de que las definiciones juegan un papel central para precisar de manera sólida y operar en diferentes contextos los conceptos matemáticos, en algunos casos las definiciones para un mismo concepto varían de un libro de texto a otro y en muchas ocasiones causan confusión entre los lectores (léase profesores y estudiantes). A título de ejemplo, se tiene el caso de la definición de la convexidad de las funciones de una variable como se identificó en algunos libros de Cálculo y Análisis Matemático más comúnmente utilizados en el nivel medio superior y superior en México. En tales textos encontramos diferencias en relación a la definición de función convexa (diferencias, en lo fundamental, en el sentido de restricciones); algunos autores asumen la noción de derivada de una función para definir el concepto de convexidad, por ejemplo: Granville (2009), entre otros, utiliza la frase de "Cóncava hacia arriba o Convexa hacia abajo" para hablar de convexidad de las funciones de una variable y además, también plantea la definición en términos de la derivada.

Por el contrario, hay quienes definen la convexidad sin recurrir al concepto de derivada, como lo indican, por ejemplo, entre otros: Spivak (2003); Bartle y Sherbert (2005), estos autores utilizan el término: "Funciones cóncavas hacia arriba“, para hablar de convexidad, en la definición que plantean no utilizan el concepto de derivada. Resulta atinado plantear que de los libros analizados, por ejemplo el Piskunov (2004) se interpreta lo convexo como lo cóncavo y viceversa. Por otro lado, la definición de convexidad en los diccionarios de matemáticas, sólo dan una idea muy vaga de lo que es una definición de convexidad para funciones. En Pisot y Zamansky (1972) encontramos la siguiente definición: Sea $f$ una función de la variable $x$ definida en un intervalo $I$. Se dirá que $f$ es convexa, si el conjunto de los puntos $(x, y)$ de $\mathbb{R}^{2}$ tales que $y \geq f(x)$ es un conjunto convexo (un conjunto $A \subseteq \mathbb{R}^{2}$, se dice que esconvexo, si dado cualquier par de puntos del conjunto, el segmento que los une está incluido en el conjunto). Mientras que Rudin (1980) define una función convexa como una función continua definida en $(a, b)$ tal que $\forall x, y \in(a, b)$ se cumple que:

$$
f\left(\frac{x+y}{2}\right) \leq \frac{f(x)+f(y)}{2}
$$

Aunque es claro que ambas definiciones son equivalente; nótese que la definición dada por Pisot y Zamansky tiene un carácter esencialmente geométrico; mientras que la dada por Rudin es puramente analítica. 
Finalmente, en Rudin (1988) se plantea una definición más general: Se dice que una función real $f$ definida en un segmento $(a, b)$, con $-\infty \leq a<b \leq \infty$, es convexa si satisface la desigualdad:

$$
\frac{f(y)-f(x)}{y-x} \leq \frac{f(z)-f(y)}{z-y}, \quad \text { siempre que } a<x<y<z<b
$$

Los textos analizados, en los cuales definen el concepto de función convexa; pudimos constatar, en primer lugar, que no hay uniformidad en la presentación de los conceptos y sus definiciones. Esta situación nos motivó a entrevistar a un grupo de cinco profesores, que imparten la unidad de aprendizaje de Cálculo Diferencial e Integral, de la Facultad de Matemáticas de la Universidad Autónoma de Guerrero, México, con la finalidad de encontrar más argumentos que nos permitieran justificar nuestra investigación.

Pregunta ¿Desde su punto de vista, cómo define una función convexa? Obtuvimos las siguientes respuestas:1) Dado un intervalo I de la forma $(\alpha, \beta),[\alpha, \beta],(\alpha, \beta],[\alpha, \beta)$, donde $\alpha$ es un número real $0-\infty, \beta$ es un número real $0+\infty$, con $\alpha<\beta$ y dada una función real $f$ definida en $A \subset \mathbb{R}$ tal que $A \supset$ (o sea, $I \subset A$ ), se dice que $f$ es una función cóncava hacia arriba (y con ello, convexa hacia abajo) si se cumple que existe un elemento $c \in$ l tal que $\alpha<c<\beta$ (es decir, si $c$ es un punto interior de $I$ ) y si $c$ es tal que:

$$
\begin{aligned}
& \forall x_{1}, x_{2}, x_{3}, x_{4}, \in I: x_{1}<x_{2}<c<x_{3}<x_{4}, \quad \Rightarrow \\
& \frac{f\left(x_{1}\right)-f\left(x_{1}\right)}{x_{1}-x_{2}} \leq \frac{f\left(x_{2}\right)-f(c)}{x_{2}-c}<\frac{f(c)-f\left(x_{3}\right)}{c-x_{3}} \leq \frac{f\left(x_{3}\right)-f\left(x_{4}\right)}{x_{3}-x_{4}} .
\end{aligned}
$$

Todo esto con $f$ continua en $l$; 2) $f: A \subset \mathbb{R} \rightarrow \mathbb{R}$ es convexa $\forall a, b \in A$, si $x \in[a, b]$, entonces $f(x) \leq \ell(x)$, donde $\ell(x)$ es una recta que pasa por los puntos $(a, f(a))$ y $(b, f(b))$, como se muestra en la Fig. 1.

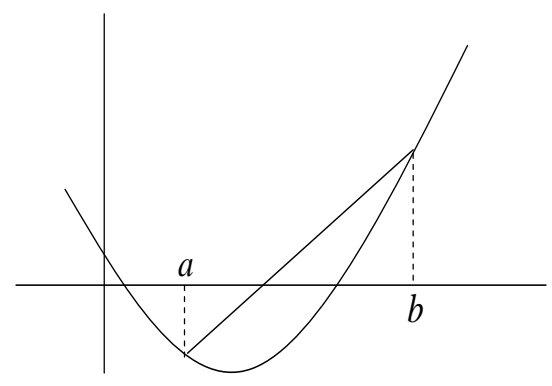

Fig. 1: Representación de región convexa [según el profesor entrevistado]

3) Como una función tal que al tomar cualquier subintervalo $(a, b)$ del dominio, la imagen del punto medio de $(a, b)$ no sobrepasa al promedio de las imágenes de los extremos del intervalo,

$$
f\left(\frac{a+b}{2}\right) \leq \frac{f(a)+f(b)}{2}
$$

4) Una función es convexa si para todo punto se puede trazar una recta tangente y la gráfica de la función siempre queda por encima de la recta tangente; 5) Una función convexa es una curva que crece y luego decrece. No estoy seguro si necesariamente debe alcanzar un punto máximo. No confundir funciones convexas con regiones convexas.

Observamos que las definiciones y terminología utilizada por los profesores son similares a la encontrada en los libros de texto más utilizados en nuestra universidad, consideramos que esto es debido a que la mayoría de estos profesores fueron formados con los textos clásicos, mencionados anteriormente, y el hecho de que en la práctica tradicional de enseñanza los docentes tiendan a hacer repeticiones textuales de las definiciones plasmadas en los libros de texto, puede obedecer, al factor de que los docentes que formaron a estos profesores incidieron en hacer prevalcer dicha práctica de enseñanza. Esta identificación sobre la definición de función convexa y de las dificultades indentificadas en los profesores, nos permiten suponer que en los estudiantes se generen confusiones y dificultades que podrían hacermás difícil el proceso de adquisición de conocimientos para ellos, en particular sobre la de definición de función convexa. 
Por otra parte, en las investigaciones en el campo de la Matemática Educativa en torno al tratamiento de las definiciones (léase Morales et al. (2014), entre otras) identifican la importancia que le dan a la definición en matemáticas y es por ello, que a partir de estudiar distintos conceptos de objetos matemáticos (familias de triángulos y cuadriláteros, ángulo recto, triángulo, conceptos del cálculo, entre otros objetos) los investigadores han identificado errores y dificultades en su tratamiento metodológico, y al mismo tiempo han intentado, a partir de proponer procesos de elaboración y construcción de definiciones, superar los errores o dificultades metodológicas asociadas con la asimilación de los conceptos en estudiantes y profesores de nivel medio y superior.

Con este antecedente, en la investigación nos planteamos el siguiente objetivo: Aportar elementos al proceso de enseñanza-aprendizaje de la Matemática a través del estudio del papel que juega el contraejemplo en los procesos de elaboración de definiciones en el contexto escolar: El caso de la función convexa. Las preguntas científicas que guiaron esta investigación fueron: 1) ¿Qué papel juegan los contraejemplos en el proceso de construcción de conocimentos en el ámbito escolar?; 2) ¿Qué importancia tiene el uso consciente de contraejemplos para el proceso de aprendizaje de los estudiantes dentro del contexto escolar de debate científico?; 3) ¿Qué papel juega el contraejemplo en el proceso de construcción de la definición de función convexa en un contexto escolar de debate científico?

\section{ELEMENTOS TEÓRICO-METODOLÓGICOS}

En esta investigación, entenderemos por concepto: El reflejo mental de una clase de objetos, procesos y relaciones de la realidad objetiva o subjetiva (o el reflejo de una clase de clases), sobre la base de sus características invariantes. Entre los conceptos se pueden considerar determinadas relaciones: Conceptos superiores, subconcepto, conceptos colaterales, conceptos disjuntos, concepto interferenciado. Por definición de concepto, entenderemos: a) Una determinación sobre lo que es un objeto, cómo se origina o cómo se reconoce; b) Una regla que establece cómo se utiliza un signo verbal; c) Una determinación o una regla, que indica o establece qué significa o debe significar un signo verbal.

Identificamos además, dos tipos de definiciones. La definición existencial: En este tipo el objeto a definir ya existe y se describe a través de sus características y la definición genética: En estas se expresa cómo puede obtenerse el objeto a definir, Zilmer (1981). Las definiciones existenciales se pueden clasificar, según el tipo de construcción del definiens (Se puede escribir una definición en forma de ecuación $X=Y$, en esta ecuación $X$ es lo que debe definirse, difiniendum, y por $Y$ entendemos lo que define a $X$, definiens): 1) El definiens se compone del concepto superior y de características significativas; 2) El definiens se compone de un número de axiomas que deben ser válidos para el objeto a definir (definición axiomática); 3) El definiens se compone de una sucesión de indicaciones recursivas (definición inductiva).

Desde la Teoría del Conocimiento, se postulan dos vías generales a través de las cuales se pueden conducir a los alumnos a la formación y definición de nuevos conceptos. La vía inductiva: Se caracteriza porque se parte de ejemplos particulares y se llega a una generalización. La vía deductiva: Se parte de la definición del concepto y mediante el análisis de ejemplos se descubre el contenido y extensión del concepto, Zilmer (1981). Tomando como justificación los argumentos anteriores, planteamos el problema de investigación en los siguientes términos: Comúnmente en los cursos tradicionales de Matemáticas los procesos de construcción de definiciones se encuentran ausentes, privando a los estudiantes de participar en el proceso de construcción de su propio conocimiento. La actividad del investigador en matemáticas subraya, en la construcción de conceptos, la importancia de la construcción de definiciones, en lo fundamental, como elemento básico operante en un determinado contexto matemático.

En este trabajo utilizamos el término "elaboración de definición" en su sentido metodológico y no entraremos en detalles asociados a elementos filosóficos sobre la discusión del término definición. A continuación realizamos un acercamiento metodológico a las siguientes cuestiones básicas para nuestra investigación: ¿Qué es una definición?, ¿qué es definir?

Comencemos por la acepción cotidiana. Etimológicamente, definir significa: delimitar lo que es de lo que no es. Este aspecto está presente en las definiciones dadas en los diccionarios y enciclopedias como se muestra en los siguientes ejemplos: El término "definir" tiene dos acepciones en el Pequeño Larousse llustrado; una es acorde al significado etimológico, es decir "determinar, delimitar con exactitud". La otra consiste en "fijar y enunciar con claridad y exactitud la significación de una palabra".

En la enciclopedia Encarta, "definición": "Proposición que expone con claridad y exactitud los caracteres genéricos y diferenciales de algo material o inmaterial. Tradicionalmente, definir es explicitar, cuando se trata de una palabra, y, cuando se trata de un ser, es asignarle un estatus; se define por género próximo y diferencias específicas" (Wikipedia llama a esto el método aristotélico de definición). Sin que la palabra 
"definición" esté, propiamente hablando, definida, aparecen dos tipos de definiciones: las definiciones intuitivas y las definiciones generativas: estas últimas consisten en dar un procedimiento de engendramiento por una consigna verbal breve, en donde el definiens regula la generación del definiendum. Los términos "definir" y "definición" de entrada parecen simples, sin embargo, las definiciones dadas en Wikipedia utilizan términos más sofisticados, sin ejemplo ilustrativo en donde "definición" es analizada como: "explicación del sentido de una palabra o la naturaleza de una cosa; ella (la definición) consiste en analizar la comprensión de una idea, por ejemplo enunciando su género próximo y su diferencia específica".

Nótese que la mayor parte de los diccionarios y enciclopedias distinguen generalmente diferentes tipos de definición sin precisar si se trata de una necesidad usual o científica, practicada o no. Estos tipos de definiciones no son analizadas en el sentido estricto del término. Subrayemos igualmente que la función de los diccionarios es habitualmente ilustrar las definiciones mediante ejemplos y raramente, o incluso nunca, mediante contraejemplos. Además, una expresión aparece recurrentemente cuando se trata de definiciones: la definición por género y diferencias específicas. En esta práctica, dos puntos que nos parecen pertinentes destacar son: 1) Los diferentes tipos de definición están dados, sin justificar las razones que los motivan, ni especificación de la disciplina a la cual se asocian: se habla de definición nominal, esencial, pero también generativa, intuitiva, analítica y genética y 2) Entrando en la Acepción matemática. En Morales et al. (2014) se trabaja sobre la base de asumir por definición: a) una determinación de qué es un objeto, cómo se origina o cómo se reconoce; b) una regla que establece cómo se utiliza un signo verbal; c) una determinación o una regla, que indica o establece qué significa o debe significar un signo verbal.

En los diccionarios de Matemáticas encontramos, en lo fundamental, las siguientes clasificaciones de definición: 1) Definición explícita: Introducción de un concepto mediante una expresión compuesta por conceptos conocidos; 2) Definición implícita: Unión de varios conceptos por medio de teoremas; 3) Definición recurrente: Se define una determinada propiedad para el número1. Además, se define cómo se deduce esta propiedad para el número $n+1$, a partir de su validez para $n$. En la misma dirección se plantea que se llama definición de un término matemático toda proposición del cual es una abreviación.

Lo que destaca en estas definiciones es que en matemáticas una de las características de la definición es su aspecto abreviativo-relacional. Encontramos también esta idea en Polya (1957) que menciona que "definir un término, es dar su significado en otros términos que se suponen conocidos: se trata de hecho de sólo definir a partir de términos ya conocidos" y Polya distingue los términos primarios de los términos derivados. En relación a las definiciones dadas por los diccionarios generales y matemáticos, precisa que: "Las definiciones dadas en los diccionarios no difieren mucho de las definiciones matemáticas por su forma exterior, pero están redactadas bajo una idea diferente" (Polya, 1957; p. 68).

Sin embargo, en matemáticas, la función de las definiciones tiene un sentido mucho más concreto que las definiciones cotidianas no sólo, según Polya (1957) porque la definición matemática crea el significado matemático, sino también su función y precisión conceptual son diferentes. En efecto, Polya enuncia que para poder eliminar un término técnico hay que conocer su definición, y así, la utilización de definiciones matemáticas conocidas permite edificar un razonamiento y verificarlo. Por otro lado, observamos que el aspecto abreviativo y la descomposición de una definición en términos primarios y/o ya definidos, es característica de toda exposición matemática teórica; tomemos el ejemplo de las obras de Rudin $(1980,1988)$. Indudablemente, Rudin es un buen ejemplo de exposición deductiva de la matemática. En las obras de este autor, el lugar de las definiciones al inicio de cada tema, con miras a abreviar el discurso es elocuente y testimonia cierta concepción abreviativa. En resumen, distinguimos dos aspectos importantes relativos a las definiciones en estos trabajos: el carácter abreviativo de una definición, y la construcción axiomática y el lugar de las definiciones en una exposición matemática.

\section{DEFINICIONES EN LA CONSTRUCCIÓN DEL CONOCIMIENTO MATEMÁTICO}

El análisis acerca de la definición en su acepción matemática pone en evidencia su importancia en un sistema axiomático pues, junto con los axiomas y los términos primarios sirven de base al razonamiento y son parte fundamental de toda la teoría matemática. Lakatos (1984) presenta un análisis notable acerca de la actividad de los matemáticos y la producción de conocimientos, situándose en un marco más amplio que el aspecto lógico o la presentación axiomática. Considera esta actividad como una interacción dialéctica entre pruebas y refutaciones, entre ejemplos y contraejemplos en medio de la cual las definiciones van evolucionando de acuerdo a las necesidades generadas por la búsqueda de una prueba. La clase de problemas en los cuales se interesa Lakatos está principalmente constituida de problemas históricos (la fórmula de Euler para poliedros, y la relación entre la convergencia de sucesiones de funciones y la continuidad con Cauchy, por ejemplo). Se trata de problemas esencialmente intramatemáticos y él pone énfasis en la fase heurística pero también en la axiomática, i. e. ¿cómo se llega a tal o cual teoría? Estos problemas no fueron resueltos en un día y la presentación del autor adopta cierta metodología en el sentido heurístico. 


\section{Método de pruebas y refutaciones}

Lakatos (1984), describe el proceso de construcción de conceptos ligado a la búsqueda de una demostración, en el que describe la evolución de diferentes definiciones, en el método de pruebas y refutaciones enuncia tres reglas heurísticas. Regla 1. Si se dispone de una conjetura, proponerse probarla o refutarla, es decir; se trata de buscar contraejemplos tanto de la conjetura (aspecto global) como de los lemas sospechosos (aspecto local). Regla 2. El descubrimiento de un contraejemplo genera un nuevo examen de la prueba y una búsqueda del lema "culpable": no se debe relegar un contraejemplo, es decir, contentarse con una relegación de excepciones. Tampoco se debe descartar una refutación como un "monstruo". Regla 3. El contraejemplo puede por lo tanto ser local (contraejemplo para un lema) o global (contraejemplo para la conjetura): se debe entonces verificar si un contraejemplo local es global y en caso de que sí, remitirse a la Regla 2.

Es en la búsqueda de una prueba, o más bien el estudio de una determinada conjetura que, según Lakatos (1984), conducen a la formación de uno o varios conceptos, y esto se traduce notablemente por la evolución de las definiciones de éstos. Así, las diferentes definiciones dadas en el transcurso de un debate científico, señalan de alguna manera la formación del concepto. Lakatos (1984), introduce entonces la terminología: definición-cero y definición generada por la prueba. Según esto, un procedimiento de construcción de definiciones corresponde a la formación del concepto el cual también proviene de la prueba.

Definiciones-cero. Constituyen un atajo de lenguaje, sin que el concepto matemático así designado esté aun definido. Estas pueden ser definiciones "de ensayo" al inicio de la investigación. Este concepto de definicióncero funciona a la par de la idea de concepto-cero. Sin embargo, ciertas situaciones pueden no recurrir a definiciones-cero, salvo cuando un concepto matemático se revela útil. Así, una definición-cero está en el origen del proceso de búsqueda. Puede ser conducida a desaparecer o a evolucionar hacia una definición generada por la prueba, este tipo de definiciones poseen una función de denominación. Lakatos, insiste, en particular, en la importancia de la evolución de una definición-cero hacia una definición generada por la prueba. Establece que las definiciones-cero poseen las cualidades siguientes: 1) Se trata de una definición que puede ser adoptada, a título de ensayo, en el origen del proceso de búsqueda, resaltando que la función primera de la prueba es determinar el dominio de validez de la conjetura y por tanto revelar el verdadero concepto y su definición. A lo más, la definición cero puede ser muy amplia y de ninguna utilidad, o muy estrecha y constituir un obstáculo que oculta ciertas partes del dominio de validez (Lakatos, 1984 p.175). Además, el problema de partida, en el origen del proceso de búsqueda y que induce definiciones-cero puede ser una fórmula, lo que será suficiente para iniciar la búsqueda. Por ejemplo, Lakatos considera que se puede iniciar a los alumnos en situaciones problemas no por definiciones sino mostrándoles un cubo, un octaedro y mostrándoles que $V-A+C=2$, y así pedirles el dominio de validez de esta fórmula. De esa manera, el investigador establece que como punto de partida, es un buen principio (Lakatos, 1984).

\section{Definiciones generadas por la prueba}

Desde el punto de vista de Lakatos, el aspecto más importante en la actividad de definición es el concepto generado por la prueba y su definición. Reiteramos que una definición generada por la prueba nace de la prueba. En relación a la formación de conceptos, se trata de hecho de encontrar un contexto problemático. El interés del estilo heurístico reside en la puesta en evidencia de la "lógica" que ha dado a luz al nuevo concepto (Lakatos, 1984, p. 168).

Lakatos utiliza el ejemplo de la prueba de Dirichlet de la conjetura de Fourier para confrontar al estilo deductivista con el estilo heurístico. En este caso, el estilo deductivista (refiriéndose al estilo empleado por Rudin en Functional Analysis (1973)) sitúa a la definición y al estudio de función de variación acotada en un capítulo sobre la integral de Riemann-Stieltjes, sin hacer el enlace entre estos dos conceptos. El autor propone en su lugar, en un estilo heurístico, cómo la integrabilidad en el sentido de Riemann-Stieltjes y la variación acotada son dos conceptos generados por la prueba surgidos de la búsqueda de la prueba de Dirichlet de la conjetura de Fourier.

En la teoría, el uso y la generación de ejemplos y contraejemplos se utilizan simultáneamente en el momento de los intercambios y conjeturas entre las personas que intervienen en el proceso de definición, en esta teoría los ejemplos y contraejemplos juegan un papel central. En actividades de determinación del dominio de validez de una conjetura o de una fórmula, se debe interrogar sobre: ¿qué efectos pueden producir los contraejemplos? Lakatos presenta el método de exclusión de monstruos, la rendición y el método de exclusión de excepciones, así como las consecuencias sobre la definición producida (reformulación, exclusión o inclusión del contraejemplo). La exposición del método de exclusión de monstruos trata de hecho de los casos dichos patológicos: según los defensores de este método, esos casos patológicos no deben ser considerados como contraejemplos, los monstruos sólo son monstruos, no contraejemplos. 
En cuanto al método de exclusión de excepciones, de manera un poco análoga al método precedente, considera que sólo existen excepciones, no contraejemplos: el problema es saber qué objeto se define (por ejemplo, ¿se definen los poliedros eulerianos o los poliedros en general?). Así, Lakatos sugiere utilizar el método de exclusión de excepciones para encontrar el dominio de validez de la fórmula de Euler en su caso preciso, pero proscribirla si sólo constituye un artificio lingüístico para salvar "agradables" teoremas. El problema reside entonces en la separación entre las excepciones y el dominio de los ejemplos. El mecanismo de utilización y de producción de ejemplos y de contraejemplos aparece, quizá de manera más sistemática en el proceso dialéctico entre la construcción del concepto y la elaboración de la prueba.

La modelización de Lakatos, muestra también cómo el proceso de matematización elimina estos contraejemplos por: exclusión de monstruos, exclusión de excepciones, incorporación de lemas, etc. Una vez que el trabajo está concluido, es decir, en la presentación clásica (axiomática) de estos resultados, el andamiaje de los contraejemplos desaparece del texto. Según Brousseau (1986) se produce una descontextualización, una despersonalización y una destemporalización de los conocimientos, lo cual constituye una de las etapas de un proceso llamado transposición didáctica. A continuación asumiremos algunos elementos esenciales de la Teoría de Situaciones Didácticas de Brousseau que permitan justificar los alcances metodológicos del "Debate Científico" propuesto por Marc Legrand, Legrand (1993).

En la construcción del conocimiento para Brousseau (1999) el término "situación" asume un papel trascendental: "Hemos llamado 'situación" a un modelo de interacción de un sujeto con cierto medio que determina a un conocimiento dado como el recurso del que dispone el sujeto para alcanzar o conservar en este medio un estado favorable. Algunas de estas "situaciones" requieren de la adquisición 'anterior" de todos los conocimientos y esquemas necesarios, pero hay otras que ofrecen una posibilidad al sujeto para construir por sí mismo un conocimiento nuevo en un proceso "genético". Brousseau (1999) plantea que una "situación didáctica" es "Un conjunto de relaciones establecidas explícita y/o implícitamente entre un alumno o un grupo de alumnos, un cierto medio (que comprende eventualmente instrumentos u objetos) y un sistema educativo (representado por el profesor) con la finalidad de lograr que estos alumnos se apropien de un saber constituido o en vías de constitución."

La Teoría de Situaciones Didácticas busca estudiar, en lo fundamental, situaciones problémicas para la apropiación del conocimiento apoyándose en enfoques constructivistas del aprendizaje (Artigue, 1994; citado en Calvo, 2001). Dicha concepción constructivista, esencialmente, tiene un sentido piagetiano del aprendizaje, concepción que es asumida por Brousseau (1986) de la siguiente manera: "El alumno aprende adaptándose a un medio que es factor de contradicciones, de dificultades, de desequilibrios, un poco como lo hace la sociedad humana. Este saber, fruto de la adaptación del alumno, se manifiesta por respuestas nuevas que son la prueba del aprendizaje." En la misma dirección la teoría postula que el profesor debe proponer un sistema de situaciones de manera que la estrategia óptima para resolver el problema aparezca de la interacciones de los estudiantes y utilizando algún aspecto del conocimiento elaborado. En otras palabras, se entiende que el alumno aprende un conocimiento cuando es capaz de adaptarse, mediante una serie de modificaciones de su estrategia, a las diferentes situaciones que se propusieron para el estudio en la adquisición del conocimiento. En ese contexto, Brousseau (1986) sostiene que hay necesidad de diseñar y organizar actividades desafiantes, afirmando que el conocimiento de interés puede, e incluso debe, ser planificado. Las "situaciones didácticas" permiten construir en los estudiantes, una "génesis artificial" de ideas matemáticas específicas cuya génesis histórica y diversos aspectos en la teoría actual son comprendidos y ya conocidos.

La Teoría de las Situaciones Didácticas según (Brousseau, 2007), pone el énfasis en sistema de preguntas y cuestiones que el docente propone al estudiante, en los conocimientos previos y nociones que estructuran sus respuestas, en un proceso complejo de estructuración-desestructuración-estructuración asociado al proceso de enseñanza-aprendizaje, mediante múltiples operaciones intelectuales y procesos mentales tales como: conjeturar, ejemplificar, abstraer, generalizar, argumentar, explicar, demostrar, deducir relaciones, entre otros. En esta dirección el estudiante aprende en relación a la actividad que realiza, por la estructuración dinámica del sistema de conocimiento, por la posibilidad de integrar nuevo conocimiento e información a las concepciones previas que posee y por la posibilidad de socializar dentro de las clases y en su propio contexto la reconstrucción de la información. Para la operacionalidad de la teoría, desde el punto de vista metodológico, la misma distingue tres importantes tipos de situaciones didácticas: Situación de acción, de formulación y situación de validación.

Aunque asumimos de forma explícita, en lo fundamental, por la importancia que reviste dentro de la Matemática Educativa los trabajos de Brosusseau sobre situaciones didácticas; cabe señalar los aportes que en la misma dirección ha desarrollado Díaz - Barriga (1996). En dichos trabajos las secuencias constituyen una organización de las actividades de aprendizaje que se realizarán con y para los estudiantes con la finalidad de crear situaciones que les permitan desarrollar un aprendizaje significativo. Otro elemento básico 
desarrollado por ambos autores y con la misma connotación dentro del proceso de enseñanza-aprendizaje y que se explicita dentro del debate científico de Marc Legrand; es la llamada institucionalización; Brousseau (1994) define su objeto de la siguiente manera: "La consideración "oficial" del objeto de enseñanza por parte del alumno, y del aprendizaje del alumno por parte del maestro, es un fenómeno social muy importante y una fase esencial del proceso didáctico: este doble reconocimiento constituye el objeto de la institucionalización."

\section{El debate científico en cursos de matemáticas}

Marc Legrand (1993) considera que una forma de abordar el conocimiento matemático es mediante la propuesta del "Debate Científico". El debate se establece entre los estudiantes a partir de situaciones problemáticas propuestas por el profesor o cuestiones y/o conjeturas que los estudiantes aportan y que puede debatirse sobre ellas a fin de construir su significado. Consideramos que los trabajos desarrollados por Legrand, son un ejemplo de la posibilidad del diseño y puesta en marcha de las ideas básicas de la teoría de situaciones didácticas y, en particular, del sistema de actividades propuestas Brousseau llamado "génesis artificial" y en este sentido se pone de manifiesto la posibilidad de crear y hacer funcionar las comunidades micro-científicas, pues en una situación mediada por el debate es propicio el trabajo con grupos o comunidades micro-científicas de estudiantes.

Dentro de las características básicas del debate científico en esta investigación se asumen las siguientes: Naturaleza y estado de los enunciados: El tipo de enunciados que se trabajan bajo el debate científico son de tipo conjetural, es decir; enunciados que pueden ser verdaderos por quien los propone, pero para la clase no tiene un carácter institucional. Este tipo de enunciados son los que posibilitan la puesta en escena del debate. Forma adoptada por los que intervienen en el debate: Por una parte es la forma en la que el alumno se posicione en relación a sus aciertos, y por otra, la elección de sus interlocutores privilegiados. Esto es, la forma en que el estudiante se dirige al profesor para manifestarse ante su aserción, o comprometerse con sus compañeros haciendo hipótesis relativas al tema en cuestión. Desde esta visión, se considera que para que haya una real comprensión de contenidos a través del desarrollo de un debate científico, es necesario considerar lo siguiente: los enunciados sobre los cuales se trabaja son de tipo conjetural, existe un contrato didáctico que legitima el hecho de que todo alumno puede someter sus propias conjeturas a la clases, los alumnos que escuchan estas conjeturas deben poder dudar de su pertinencia o veracidad.

\section{El papel del alumno}

Es importante hacer una negociación didáctica con la intención de conducir al alumno a considerar que va a adquirir conocimientos de manera diferente a como quizás lo ha venido haciendo a lo largo de su formación, ciertamente no es tan sencillo por la forma en cómo se relaciona normalmente con sus compañeros, sin embargo, es factible insistir que va a aprender escuchando y analizando de forma sutil los propósitos de la interacción maestro-alumno, y que además tendrá oportunidad de convencer acerca de lo bien fundamentado de sus aserciones, sin necesitar de la intervención previa del profesor.

\section{El papel del profesor}

El profesor debe manejar simultáneamente una triple función: epistemológica, didáctica, y social. Es decir, debe estar consciente en todo momento de lo que es matemáticamente puesto en discusión, debe permitir que el debate se desvíe al ritmo de su propia comprensión o de los mejores alumnos; para esto, anota lo que se dice con el fin, por una parte, de hacer más lenta la velocidad de los intercambios de opiniones, y por otra parte, construir una lista de ideas fuertes (verdaderas o falsas), con la finalidad de que cada uno pueda discutir acerca de ellas. De esta manera el profesor permanece neutro acerca de lo que se dice, y organiza una memoria de la clase, y considerar que el aspecto social es muy importante, por la necesidad de que alguien conduzca al grupo, con el fin de evitar que el debate se hunda en un foro en donde cada uno grite. Según Legrand (1993) el contraejemplo puede ser una herramienta de regulación del debateque se haya generado.

\section{La apuesta del debate}

Es importante mencionar que el objetivo del debate no es el descubrimiento de propiedades matemáticas nuevas, sino, en lo fundamental, la toma de sentido respecto a teorías bien establecidas. Aquí se trata de descubrir la significación de resultados y de apropiarse de los métodos de razonamiento que confieren cierta independencia de pensamiento. Por otra parte, un elemento esencial del contrato didáctico es: hacer compartir la hipótesis de que, si el cuestionamiento interno de la clase es importante, va a permitir la vida en la duración de la clase, de problemáticas científicas indispensables para la constitución del sentido. 


\section{El tratamiento de las definiciones}

Los objetos que manipulamos en matemáticas, incluso cuando están transpuestos de una realidad sensible, son sin embargo, objetos construidos, y no se puede discutir sobre ellos matemáticamente si su definición no es clara; pero esto es desde el punto de vista del discurso del profesor, y los alumnos, sobre todo aquellos que tienen dificultades, no tienen en general que hacer tal discurso. El tratamiento de las conjeturas intuitivas provoca tomar decisiones muy radicales que obligan al final de un tiempo a plantear la siguiente cuestión: ¿todos estamos hablando de lo mismo? En el debate para la elaboracion de una definición los actores tienen muchas oportunidades de ser escuchados, incluso por aquellos que evaden todo lo que tenga un aspecto formal, porque la definición se transforma entonces en el único medio racional de deshacerse de un malentendido.

Tipos de situaciones manejadas por el debate: a) Las situaciones de introducción a un nuevo concepto. Cuando se habla de debate en la clase, se piensa principalmente en proponer trabajar situaciones problemáticas que permiten la introducción de un concepto. El debate científico puede favorecer en la búsqueda de significados de los conceptos que conforman parte de la estructura de la matemática. Si bien es cierto que, no para todos los conceptos matemáticos existe una relación con un problema real; a nuestro ver pueden plantearse situaciones que provoquen y propicien en los estudiantes interés en la discusión, análisis, y definición de los conceptos, utilizando como herramienta el contraejemplo; b) El debate sobre conjeturas. Estos debates se producen lo más a menudo cuando el curso ha emprendido la fase de desarrollo de una teoría. Cuando se han adquirido elementos necesarios que verifican casos particulares, nociones de conceptos básicos que permiten estructurar enunciados (conjeturas), cuando se establecen relaciones entre nociones similares lo cual provoca el planteamiento acerca de que si una es caso particular de la otra o viceversa, a partir de operar con objetos matemáticos que satisfacen ciertas propiedades, a partir de hechos que confirman o verifican casos particulares, cuya cantidad y variedad afectan el grado de convencimiento, a partir de intuiciones erróneas que tienen los estudiantes acerca de las nociones que los conducen a la impresión de que un terminado resultado puede ser verdadero; c) Debates espontáneos. Un tipo de debate enmarcado en la propuesta general del "debate científico", es llamado debate espontáneo. Este debate surge, cuando se devuelven las preguntas en forma de conjeturas en la microsociedad científica o grupo de clase el cual da la responsabilidad de responder bajo tres formas: verdadero, falso, otro; donde "otro" significa "no puedo" o "no quiero responder bajo la forma verdadero/falso".

Esta devolución de las preguntas a los estudiantes en forma de conjeturas, pocas o raras veces se han tratado a profundidad, y generalmente las preguntas hacia el profesor se han pensado como aquellas donde los alumnos o bien no han escuchado nada o bien, están distraídos de lo que en ese momento se estaba poniendo en juego. Considerar los argumentos que los estudiantes hacen acerca del contenido que se está tratando, posibilita la introducción de otros conceptos que en ese momento no son objeto de discusión, pero que además el profesor supone que los estudiantes ya dominan; entonces se considera pertinente hacer un paréntesis, para dedicarle tiempo al debate sobre este tipo de conceptos y posteriormente retomar el tema principal.

La institucionalización es el momento en que el profesor sale totalmente de su neutralidad epistemológica para etiquetar, entre los resultados estudiados, aquellos que serán ciertamente reutilizados: aquellos que son verdaderos se transforman en teoremas del curso, y aquellos que son erróneos pero que corresponden a modos de pensamiento muy "naturales" son referenciados como tales. En este contexto, el profesor reafirma por lo tanto que no es tiempo perdido producir resultados falsos y darse cuenta de ello, y que es un verdadero conocimiento científico saber que tal razonamiento, tal método, tal resultado no desemboca en tal o cual situación. Una parte importante de los conocimientos institucionalizados es por lo tanto de naturaleza muy diferente de aquellos que figuran tradicionalmente en un curso, puesto que son meta-conocimientos. Una de las apuestas del debate es por lo tanto hacer descubrir a cada uno que a partir del momento en el que no se inhiben sus facultades imaginativas y creadoras (por ejemplo declarándose inaptos a la reflexión o diciéndose que no sirve para nada buscar lo que ha sido ya encontrado), se tienen ideas personales, pero que una idea científica espontánea no es por así decirlo jamás inmediatamente satisfactoria y totalmente exacta; ésta debe ser "trabajada" y sometida a la búsqueda de pruebas y de contraejemplos.

En esta fase, el profesor debe claramente indicar lo que es exacto y lo que no lo es, su rol es sobre todo no poner en vanguardia a los alumnos que habían encontrado de golpe la buena solución y despreciar la actitud de aquellos que habían cometido errores. Le incumbe, por el contrario, mostrar que la introducción de proposiciones o de razonamientos erróneos por el juego de la crítica con la sola vista del resultado final.

\section{Relación de los elementos teóricos y metodológicos}

En el proceso de construcción de la definición de un determinado concepto; las pruebas y las refutaciones juegan un papel central y en este sentido, el contraejemplo sirve de base y/o herramienta mediadora y de 
control del proceso asociado a la formación conceptual. El mismo adquiere fuerza y sentido metodológico en su relación con la validez de las conjeturas, de las argumentaciones, de las demostraciones, de las condiciones lógicas, como medio para la toma de decisión, entre otros usos. A medida que la refutación de conjeturas o proposiciones mediante el contraejemplo permiten la evolución del proceso de construcción de la definición, y una vez que el trabajo está formalizado, es decir, en la presentación clásica (axiomática) de estos resultados, el andamiaje de los contraejemplos desaparece del texto y es así, como se presenta este conocimiento (acabada e inmutable) para su tratamiento en la escuela. De esto último, se establece que se ha producido una descontextualización, una despersonalización y una destemporalización de los conocimientos, en el proceso de transposición didáctica.

En el proceso de transformación de la génesis del saber al saber sabio, la historia de los saberes es eliminada completamente, cuando son borradas las sucesiones de dificultades y preguntas, los ejemplos y contraejemplos y las innumerables discusiones generadas que provocaron la posibilidad de construir y definir los conceptos fundamentales que hoy día se plasman y tratan en los textos. Una vez que el conocimiento es presentado mediante definiciones, teoremas, axiomas, etc.; el profesor debe recrear escenarios que posibiliten la recontextualización, repersonalización y la retemporalización a fin de que el estudiante se apropie de ese conocimiento en un contexto significativo, él debe buscar la forma de crear en la clase una microsociedad científica, donde el estudiante vuelva a contextualizar, personalizar, temporalizar ese saber, con la finalidad de que identifique su producción con el saber que opera en la actualidad. Este proceso se muestra en la Fig. 2.

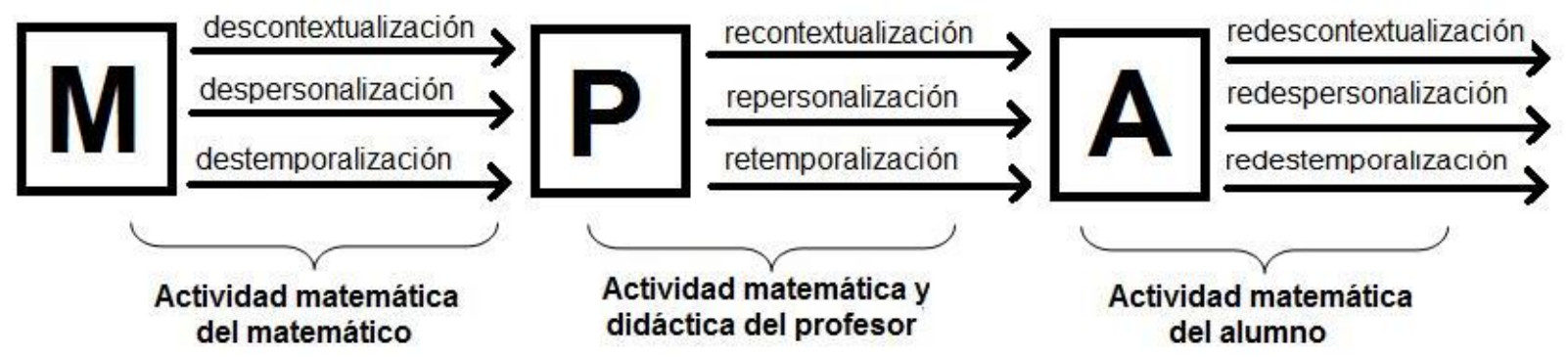

Fig. 2: Etapas de la transposición didáctica.

La teoría de situaciones didácticas busca estudiar todas aquellas situaciones de apropiación del conocimiento a partir de la adaptación del estudiante al medio donde se construye un saber. En este trabajo, defendemos el criterio que la actividad esencialmente asociada al estudiante se pone de manifiesto en la etapa de "Actividad matemática del alumno", mientras que la intervención del profesor-investigador consiste en crear las condiciones para favorecer la "Actividad matemática y didáctica" ver Fig. 2. Consideramos que la instauración de un debate científico resulta un elemento de carácter trascendental para la recreación de escenarios propicios que favorecen los procesos de construcción, en este caso, del concepto de función convexa.

\section{METODOLOGÍA}

La secuencia didáctica utilizada en la investigación fue tomada de una ingeniería didáctica diseñada para la introducción de la noción de convergencia de sucesiones de funciones (Robinet, 1992). En particular, se trabajó con la primera actividad propuesta en la primera etapa del diseño de la ingeniería didáctica, consistente en graficar trece sucesiones de funciones y determinar el comportamiento de dichas gráficas en un proceso infinito, ver Tabla 1.

La justificación del tratamiento de esta actividad, radica en el hecho de que el concepto de convergencia y de convergencia uniforme de sucesiones de funciones engloba conceptos fundamentales del Cálculo y del Análisis Matemático. Por mencionar algunos de estos conceptos: límite, continuidad, derivada, criterios de convergencia de series, determinación de dominios de definición de funciones, variación de funciones: crecimiento y decrecimiento, máximo y mínimo relativos, concavidad y convexidad, punto de inflexión, entre otros.

Dada la diversidad en los tipos de gráficas, durante el proceso de análisis por los estudiantes; se previó el surgimiento de conflictos entre ciertas nociones que se involucran en el proceso de graficación y de manera lógica se instauraron diversos debates en torno a esta actividad, en particular, la atención se centró en las dificultades relativas a la noción de función convexa y se intentó en el transcurso del desarrollo de las actividades, llevar a cabo un proceso de elaboración de la definición de esta noción. 
Tabla 1: Sucesiones de funciones propuestas por Robinet (1992).

\begin{tabular}{|c|c|c|c|c|c|}
\hline \multicolumn{2}{|c|}{$\forall n \in \mathrm{N}$ y $\forall x \in \mathrm{R} f_{n}(x)=\frac{x}{n}$} & \multicolumn{2}{|c|}{$\forall n \in \mathrm{N}$ y $\forall x \in \mathrm{R} \quad f_{n}(x)=\frac{x}{1+n^{2} x^{2}}$} & $\forall n \in \mathrm{N} y \forall x \in \mathrm{R}\{$ & $\begin{cases}f_{n}(x)=f_{n}(-x) & \text { para } x \in(-\infty,+\infty) \\
f_{n}(x)=n x & \text { para } x \in\left[0, \frac{1}{n}\right] \\
f_{n}(x)=1 & \text { para } x \in\left[\frac{1}{n},+\infty\right)\end{cases}$ \\
\hline$\forall n \in \mathrm{N}$ & $\begin{cases}f_{n}(x)=0 & \text { para } x \in(-\infty, 0] \\
f_{n}(x)=x & \text { para } x \in\left[0, \frac{1}{n}\right] \\
f_{n}(x)=\frac{1}{n} & \text { para } x \in\left[\frac{1}{n},+\infty\right)\end{cases}$ & \multicolumn{2}{|c|}{$\forall n \in \mathrm{N} y \forall x \in \mathrm{R} \quad f_{n}(x)=\frac{x^{2 n}}{1+n x^{2 n}}$} & \multicolumn{2}{|c|}{$\forall n \in \mathrm{N}$ y $\forall x \in \mathrm{R} \quad f_{n}(x)=\frac{1}{1+n x^{2}}$} \\
\hline$\forall n \geq 2$ y $\forall x \in \mathrm{R}$ & $\mathrm{R} \begin{cases}f_{n}(x)=0 & \text { para } x \in(-\infty, 0) \\
f_{n}(x)=\frac{x}{n} & \text { para } x \in[0,1] \\
f_{n}(x)=\frac{n-x}{n(n-1)} & \text { para } x \in[1,+\infty)\end{cases}$ & $\forall n \in \mathrm{N}$ y $\forall x \in \mathrm{R}$ & $\left.\begin{array}{ll}f_{n}(x)=0 & \text { para } x \in(-\infty, 0] \\
f_{n}(x)=n x & \text { para } x \in\left[0, \frac{1}{n}\right] \\
f_{n}(x)=-n x+2 & \text { para } x \in\left[\frac{1}{n}, \frac{2}{n}\right] \\
f_{n}(x)=0 & \text { para } x \in\left[\frac{2}{n},+\infty\right.\end{array}\right]$ & $\forall n \in \mathrm{N}$ y $\forall x \in \mathrm{R}$ & $\begin{cases}f_{n}(x)=f_{n}(-x) & \text { para } x \in(-\infty,+\infty \\
f_{n}(x)=n^{2} x & \text { para } x \in\left[0, \frac{1}{n}\right] \\
f_{n}(x)=n & \text { para } x \in\left[\frac{1}{n},+\infty\right)\end{cases}$ \\
\hline$\forall n \in \mathrm{N}$ y $\forall x \in \mathrm{R}$ & $\begin{cases}f_{n}(x)=0 & \text { para } x \in(-\infty, 0] \\
f_{n}(x)=n^{2} x & \text { para } x \in\left[0, \frac{1}{n}\right] \\
f_{n}(x)=-n^{2} x+2 n & \text { para } x \in\left[\frac{1}{n}, \frac{2}{n}\right] \\
f_{n}(x)=0 & \text { para } x \in\left[\frac{2}{n},+\infty\right)\end{cases}$ & $\forall n \in \mathrm{N}$ y $\forall x$ & $\in \mathrm{R} f_{n}(x)=\frac{x^{n}}{n}$ & $\forall n \in \mathrm{N}$ y $\forall x \in$ & $\mathrm{R} f(x)=\frac{x \sqrt{n}}{1+n x^{2}}$ \\
\hline$\forall n \in \mathrm{N}$ y $\forall$ & $x \in \mathrm{R} \quad f_{n}(x)=\frac{x^{2 n}}{1+x^{2 n}}$ & & & & \\
\hline
\end{tabular}

\section{Consideraciones metodológicas}

La investigación se llevó a cabo en las siguientes etapas: (i) Elección de una estrategia didáctica; (ii) Elección de la secuencia didáctica; (iii) Elección del contenido a trabajar; (iv) Recopilación y tratamiento de la información; y (v) Población objeto de estudio.

Primera Etapa. Elección de una estrategia didáctica que permitió la producción de argumentaciones y contraargumentaciones: fue el debate científico en cursos de matemáticas. Desde nuestro punto de vista, el debate científico en cursos de matemáticas es una estrategia idónea para poner en acción un proceso de construcción de definiciones en colectivo, en la cual se busca la colaboración y participación de todos los involucrados en este proceso, esta propuesta se instaura, en lo fundamental, a partir de situaciones problemáticas.

Segunda Etapa. Elección de la secuencia didáctica. Hemos tomado nuestra secuencia didáctica diseñada para la introducción de la noción de convergencia de sucesiones de funciones (Robinet, 1992) porque su estructura y diseño favorece la aplicación y, en su caso, la discusión de diferentes conceptos básicos estudiados en los cursos anteriores. La diversidad de situaciones representadas va a permitir la aparición de ciertos conflictos cognitivos entre las nociones y conceptos involucrados en el proceso de graficación, y mismos que se instaurarán en los llamados Debates Espontáneos según la terminología de Legrand (1993).

La secuencia se trabajó con los estudiantes organizados en grupos de tres o cuatro. Este trabajo en grupos es fundamental en esta situación por las siguientes razones: 1) La situación de debate científico está constituida de un tipo de tareas que está ausente de las estrategias didácticas usuales: por lo tanto es necesario organizar interacciones, tanto para favorecer la devolución de la situación ante los estudiantes como para recopilar datos para nuestra investigación; 2) Finalmente, trabajando en grupos los estudiantes estarán más cómodos tendrán mayor seguridad e inventiva, pues la diversidad de sus conocimientos permitirá al agrupo apoyarse sobre nociones más ricas. En otras etapas se les pedirá a los estudiantes trabajar solos, para poder controlar lo adquirido por cada uno acerca de la noción. 
Tercera Etapa. Elección del contenido a trabajar. Se trabajó con la noción de función convexa, en lo esencial, por la importancia que reviste dicho concepto como base de temas esenciales de la Matemática Superior tales como: Teoría de Funciones, Topología y Teoría de las Desigualdades y por la relación operacional que guarda con los conceptos de límite, continuidad, derivada e integral. Cabe destacar, además que el tema de función convexa surgió a manera de debate espontáneo. Particularmente, se estudió el proceso de construcción de la definición de función convexa, y se observó el papel que desempeña el contraejemplo en ese proceso.

Cuarta Etapa. Recopilación y tratamiento de la información. Con el objetivo de registrar las reacciones, interacciones, diálogos y discusiones; se distribuyeron en cada equipo grabadoras de voz digitales. Las exposiciones fueron registradas mediante una cámara de video la cual le daba preferencia al representante de cada equipo que exponía los resultados producto de las discusiones colectivas y a la persona que previamente solicitaba la palabra para intervenir con alguna opinión. Se registraba también lo escrito en el pizarrón. Para el análisis de la información se hizo necesario transcribir detalladamente lo registrado en las grabadoras y en la cámara de video, respetando, en todo momento, el lenguaje utilizado por los estudiantes. Se procedió entonces al análisis y síntesis de la información.

Quinta Etapa. Población objeto de estudio. La población de estudiantes con los cuales se trabajó, estuvo formada por veinticuatro alumnos del cuarto semestre de la Licenciatura en Matemáticas de la Universidad Autónoma de Guerrero, en ese momento estaban cursando la materia de Cálculo IV. Previo a la experimentación, los estudiantes estaban por iniciar el estudio del tema de sucesiones de funciones, por lo que este fue el escenario que utilizamos para realizar nuestra investigación. Otro dato relevante es que dichos alumnos han abordado ya los métodos para el análisis de funciones empleando la primera derivada para determinar el sentido de variación y los extremos, y la segunda derivada para determinar la convexidad, concavidad y puntos de inflexión. En teoría los estudiantes debieran tener dominio de las nociones que se involucran en estos procesos, por ejemplo, debiera estar claro para ellos qué es una función convexa y saber qué relación tiene con la derivabilidad y la continuidad, pero como se pudo observar en la experimentación, esto no es así.

\section{RESULTADOS}

Se presentan los resultados de la investigación, destacándole las respuestas, preguntas, interacciones y situaciones similares, entre profesor - estudiante y viceversa.

\section{Irrupción sobre el concepto de convexidad en un debate espontáneo}

El tema de la convexidad hace su irrupción en el debate en el momento en que se están exponiendo las gráficas por los estudiantes organizados en equipos de tres, de las sucesiones de funciones. Específicamente se está tratando el caso de la sucesión número 7, ver Fig. 3.

$$
\forall n \geq 2 \text { y } \forall x \in \mathrm{R} \begin{cases}f_{n}(x)=0 & \text { para } x \in(-\infty, 0) \\ f_{n}(x)=\frac{x}{n} & \text { para } x \in[0,1] \\ f_{n}(x)=\frac{n-x}{n(n-1)} & \text { para } x \in[1,+\infty)\end{cases}
$$

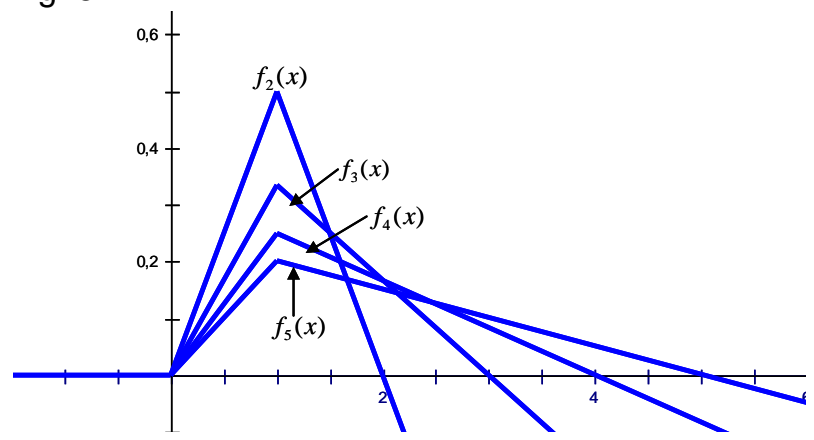

Fig. 3: Forma analítica y gráfica de la sucesión de funciones.

Un debate espontáneo. La inquietud surge a partir de la pregunta planteada por uno de los participantes: Estudiante 1(E1): ¿No existen puntos de inflexión?

[...]

Estudiante 2 (E2): No, porque en primera la función es lineal. Los puntos de inflexión están determinados al hacer la derivada. Ahí la segunda derivada sería cero, porque una función lineal su derivada es constante, si yo la vuelvo a derivar me va a dar cero. Ahora tú igualas la segunda derivada a cero para encontrar los puntos donde...este....se dice que hay puntos de inflexión, entonces te va a dar cero igual con cero nada más.

Se observó que E2 describió de una manera precisa y para un caso particular el algoritmo que sirve para encontrar los puntos de inflexión de una función. En principio para E2 y para todos los participantes en el debate debieran estar claros los conceptos que se involucran en este procedimiento, a saber el concepto de 
función cóncava, función convexa y punto de inflexión. En efecto, los estudiantes han abordado ya estos contenidos en sus cursos anteriores de cálculo. La reacción que E1 tiene hace sospechar que no es así, por lo que el PROF plantea la siguiente pregunta:

PROF: A ver creo que no estamos hablando de lo mismo ¿Qué es un punto de inflexión? ¿Qué es un punto de inflexión E1?

E1: Es donde cambia la función de...es donde hay un cambio pues...

PROF: Cambio en qué sentido ¿Qué tipo de cambio?

E1: Que crece y después decrece ¿No?

PROF: O sea que va creciendo y después va decreciendo, ahí hay un punto de inflexión, ¿Estamos de acuerdo en eso? ¿Sí?

[Se genera un debate en el que algunos dicen que sí y otros que no. Varios hablan al mismo tiempo]

PROF: Creo que no está claro eso de los puntos de inflexión.

E2: PROF, ahí se maneja el término concavidad de una curva.Donde hay ese cambio hay un punto de inflexión.

PROF: Y en este caso tenemos una gráfica ¿De qué tipo? ¿Cóncava o convexa?

[Varios contestan convexa]

PROF: Tenemos una gráfica ¿Cóncava o convexa en este caso?

[Se escuchan varias opiniones]

La diversidad de opiniones manifestadas en esta parte del debate pone de manifiesto las dificultades que los estudiantes tienen en torno a las nociones de punto de inflexión, de convexidad y de concavidad de una función. En algunos casos las opiniones son contradictorias y los partidarios de una u otra posición parecen dispuestos a involucrarse en una discusión en la que se diluciden los significados de las nociones.

\section{Definiciones que emergieron}

Una vez que el problema está planteado, aprovechando una inquietud de E1, fue necesario hacer surgir las diferentes concepciones que los estudiantes tienen acerca de la convexidad para analizar si es posible hacerlas evolucionar hasta acercarse a alguna de las definiciones establecidas en los textos de matemáticas. Se observó que la discusión acerca de la convexidad surge en lo que Legrand llama un debate espontáneo, Legrand (1993). Con esta intención se plantea la siguiente pregunta.

PROF: ¿Qué significa que una función sea cóncava o convexa?

[Hay unos segundos de discusión].

Primera definición.E1. Cuando la gráfica se mantiene en el mismo plano y no sale de ahí.

El lenguaje utilizado en esta definición dada por E1 es muy ambiguo, por lo que hay necesidad de explorar un poco más en su significado. Además, se observó que esta definición se aleja mucho de las definiciones manejadas en los textos. Es el motivo por el cual el PROF pidió a ES que ilustre su definición con un ejemplo gráfico para que ahí se identifiquen los diferentes elementos utilizados.

PROF: A ver dibújanos una gráfica que, desde tu punto de vista, sea cóncava y una convexa, en el pizarrón. La idea que tú tienes. Para que nos expliques la definición que estás dando.

$[\ldots]$

[E1 pasa al frente y traza un sistema de coordenadas]

E1: Yo tengo entendido que una función es convexa cuando está en este semiplano [señala el primero y cuarto cuadrante]. Bueno viendo esa gráfica... podemos decir que ésta es convexa [hace un trazo en el pizarrón, análoga a la siguiente construcción, Fig. 4].

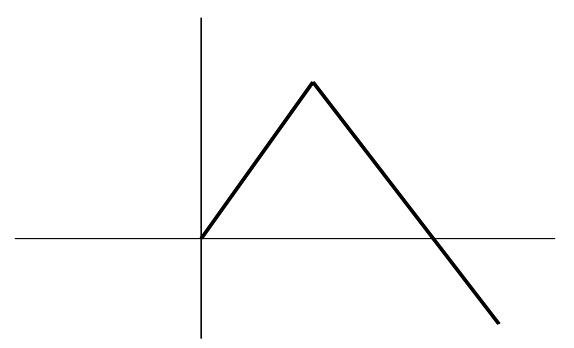

Fig. 4: Curva convexa según E1.

Una primera interpretación dada al dibujo podría hacer pensar que E1 considera que una función convexa es aquella que "abre hacia abajo" acorde con lo expuesto en Piskunov, Puskinov (2004). Esto último no está fuera de lugar, ya que los estudiantes que participaron en este debate usaron como uno de los textos 
principales para sus cursos de cálculo I y cálculo II el texto de Piskunov, es lógico que en la mente de ellos tengan esa concepción de la convexidad. Sin embargo, con el objeto de profundizar en la concepción de ES, el PROF le hace la siguiente solicitud:

PROF: Supongamos que dibujas esa misma gráfica del lado izquierdo del eje de las y. A ver dibújala, exactamente la misma así como está.

[E1 dibuja lo que se le pide, ver Fig. 5]:

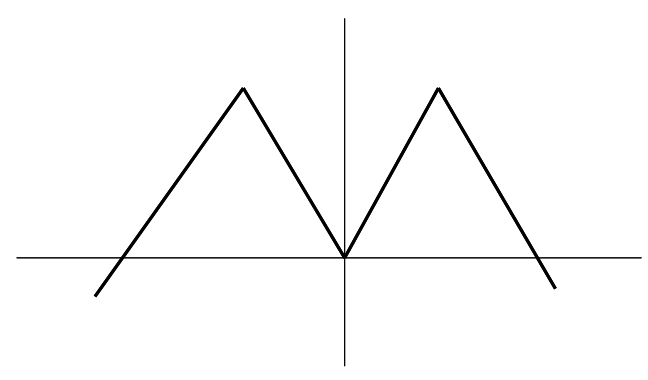

Fig. 5: Gráfica simétrica respecto al eje $y$.

PROF: ¿Esa cosa es cóncava y convexa?

E1: Sería cóncava de aquí para acá [señala del origen hacia la izquierda]...es que no sé.

PROF: Ahora dibuja una que no sea convexa...

[E1 traza una curva como la siguiente, ver Fig.6]:

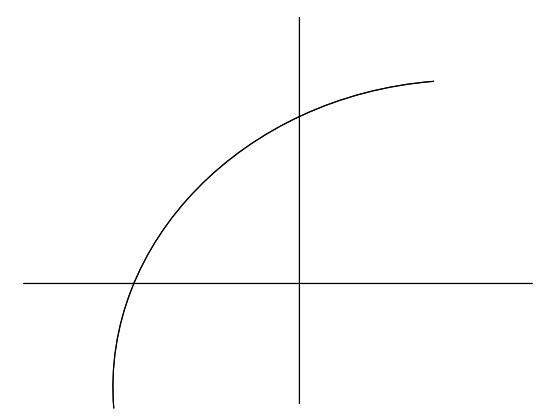

Fig. 6: Curva no convexa, según E1.

PROF: Esa no sería convexa y según tu definición es porque en ese caso cambia del plano negativo y pasa al plano positivo.

E1 asiente.

Estos dos ejemplos son acordes con lo expresado verbalmente por E1. Por tal motivo, consideramos que no es posible que esta definición ingenua evolucione para acercarse a la definición establecida. En consecuencia, esta definición se desechó al surgir otras definiciones en torno a la cuales se generaron nuevas discusiones.

En lo que sigue, solo se muestran las aproximaciones a la definición de función convexa que emergieron, producto del debate y en el que la formulación de los contraejemplos favoreció la evolución y/o la refutación en algunos casos de propuestas de definiciones.

Segunda definición. E2. Cuando derivo encuentro los puntos de inflexión, entonces tomo los intervalos del dominio de mi función y ya en ese intervalo analizo la primera derivada. Si la primera derivada en ese intervalo sufre un cambio de positivo a negativo, entonces yo puedo afirmar que mi... este... en ese intervalo la curva es convexa. $Y$ si en ese intervalo la derivada cambia de negativo a positivo, yo puedo afirmar que en ese intervalo la curva es cóncava. Yo así lo haría.

Esta definición es generada, en lo fundamental, por una intuición errónea, que tiene el estudiante, en relación a la convexidad. En efecto, para las funciones, que comúnmente se muestran, como ejemplos, en los textos, cuyos máximos se pueden calcular mediante la derivada, en una vecindad de los máximos las curvas son convexas. Evidentemente, este es sólo un caso particular de la noción de función convexa pero consideramos que mediante sugerencias críticas pudieron irse eliminando restricciones para aproximarla a alguna de las definiciones establecidas. En la terminología de Lakatos, esta definición puede fungir como una definición cero. 
Tercera definición.E2. Fíjese, lo que yo le decía era, en términos de funciones crecientes y decrecientes, yo tendría que mi función crece y de repente decrece podría ser una opción para tomar que es convexa, y cuando mi función va decreciendo en ese intervalo y de repente crece pues la puedo tomar como cóncava.La idea central en la que se basa esta definición es similar a la anterior sólo que ante la crítica de que la definición depende de la derivabilidad de la función la enuncia en términos de la monotonía. Esta definición pudo haberse criticado con un contraejemplo pues en realidad este hecho no caracteriza ni implica a la convexidad.

Cuarta definición. E3. Para las convexas, la tangente siempre va a estar por arriba, y para las cóncavas la tangente siempre va a estar por debajo, de la función... de la curva. Recuérdese que esta definición es la dada por Piskunov. Sin embargo es una definición restringida sólo al dominio de las funciones derivables. Sin embargo es posible hacerla evolucionar, al igual que la segunda.

Quinta definición. E4. Profesor, yo encontré una definición en un diccionario de matemáticas. Dice que una figura es convexa, si tomando dos puntos del interior de la figura y trazamos un segmento que une dos puntos de la figura, no se sale de la figura, esté contenido en ella. Esta definición se presenta en la Fig. 7.

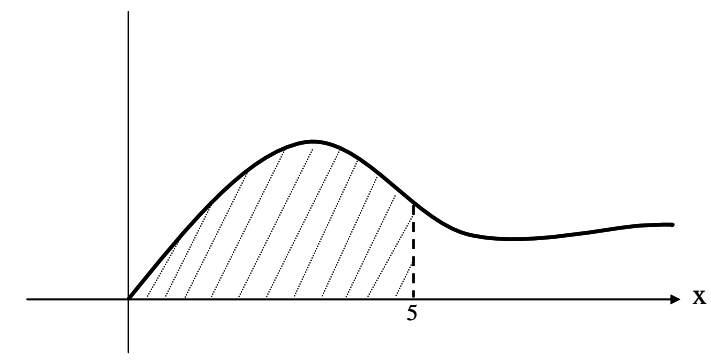

Fig. 7: Región de convexidad [según definición en diccionario de matemáticas]

E5. Ahora sí, con esa definición (que dio E4) de convexidad... Entonces, vamos a ver, si yo tomo dos puntos cualesquiera y los uno, ese segmento siempre va a pertenecer a la figura. Esta definición construida entre dos participantes es la definición cero que, por la verificación mediante ejemplos y la crítica mediante contraejemplo se hace evolucionar hacia la definición seis. Reiteremos que pudo haber evolucionado aproximándose a la definición dada en Pisot y Zamansky, Pisot (1972).

Sexta definición. E2. No profesor. Si yo tomo dos puntos de un intervalo pero que pertenezcan a la curva, si yo los uno, y si... la curva queda por encima de la recta que une esos dos puntos, se dice que es... convexa. Y si pasa por debajo la curva de la recta esa, entonces es cóncava. Se llega a esta definición después de que la quinta es puesta a prueba mediante ejemplos y finalmente desechada mediante un contraejemplo.

Séptima definición. E6. Y ya entonces para todo $x$, como ya dio la definición E3, o sea que esta sea $g(x)$ [se refiere a la curva] y esta que sea $f(x)$ [se refiere a la recta que une a los puntos] y ya a partir de ahí, si $f(x)$ es menor que $g(x)[f(x) \leq g(x)]$. Finalmente esta definición se obtiene por una solicitud de mayor precisión en el lenguaje utilizado en la definición seis. Es evidente que esta última definición no es, en términos estrictos, exactamente la definición de una función convexa. Sin embargo consideramos que está muy próxima a ella. Esta definición puede enunciarse de manera más precisa en la etapa de institucionalización con la ventaja y la convicción por parte de los participantes en todo el debate, de que las discusiones tenidas a lo largo del proceso habrán preparado el terreno para dotar de sentido a la definición formal del concepto.

En la Tabla 2 se indica el número de alumnos que aportaron propuestas sobre una aproximación a la definición de función convexa; presentadas anteriormente, indicando además, si éstas son o no refutables, y si evolucionan o no.

Tabla 2: Alumnos que aportaron propuestas afines a la definición

\begin{tabular}{|l|c|c|c|c|}
\hline Definiciones & $\begin{array}{c}\text { No. de alumnos cuya } \\
\text { respuesta es afín }\end{array}$ & $\begin{array}{c}\text { No. Alumnos que no } \\
\text { dieron respuesta }\end{array}$ & $\begin{array}{c}\text { Se refuta } \\
\text { la respuesta }\end{array}$ & Evoluciona \\
\hline Definición 1 & 18 & 6 & $\mathrm{Si}$ & $\mathrm{No}$ \\
\hline Definición 2 & 16 & 8 & $\mathrm{No}$ & $\mathrm{Si}$ \\
\hline Definición 3 & 16 & 8 & $\mathrm{No}$ & $\mathrm{Si}$ \\
\hline Definición 4 & 17 & 7 & $\mathrm{No}$ & $\mathrm{Si}$ \\
\hline Definición 5 & 17 & 7 & $\mathrm{No}$ & $\mathrm{Si}$ \\
\hline Definición 6 & 18 & 6 & $\mathrm{No}$ & $\mathrm{Si}$ \\
\hline Definición 7 & 18 & 6 & $\mathrm{No}$ & $\mathrm{Si}$ \\
\hline
\end{tabular}




\section{DISCUSIÓN FINAL}

A lo largo de la actividad desarrollada, se observó que es factible poner en funcionamiento un proceso de elaboración de definiciones en el contexto escolar y que en este proceso, los contraejemplos pueden ser utilizados adecuadamente para criticar las definiciones propuestas por los que participan en el debate y hacerlas evolucionar, aproximándolas a las definiciones que se usan en los textos escolares de matemáticas. Consideramos que el debate científico en cursos de matemáticas es un escenario propicio para un procedimiento de esta naturaleza. En la misma dirección, mediante la investigación, se puso de manifiesto que el contraejemplo es un recurso básico y una herramienta mediadora; que favorece la crítica, la refutación y afinación de las propuestas de definición, en este caso, de función convexa, mismas que emergieron en una situación de debate científico, y de esa forma se hizo evolucionar el proceso hasta aproximarla a la definición formal que usualmente utilizan los textos escolares.

El desarrollo de clases con "debate científico" favorece, mediante la discusión en torno a problemáticas que involucran los conceptos, la formulación de contraejemplos, la comprobación de casos particulares, el rechazo de conjeturas, entre otros. Hacer una reconstrucción del concepto, favorece el significado preciso, su aprendizaje y su utilidad en la misma matemática y en situaciones reales. Por otro lado, a lo largo del debate aparecieron también los disfuncionamientos previstos en el planteamiento de nuestro problema, a saber, el impacto que tiene la diversidad de definiciones y terminología existente en relación a la noción de convexidad, a pesar de que en matemáticas se acepta de manera generalizada que la precisión en las definiciones juega un papel muy importante en esta disciplina. A nivel de beneficios de la estrategia de debate, se observó cómo los participantes se comprometían con la defensa de sus respectivas posturas y cómo, al final de la experimentación relativa a la noción de función convexa, manifestaron inquietudes por propiedades más profundas de las nociones involucradas en el debate. Específicamente, lo referente a la relación entre la derivabilidad y la convexidad de funciones así como la existencia de funciones continuas convexas en ningún intervalo.

Por último, esperamos que este trabajo contribuya a llamar la atención sobre las definiciones que se utilizan en el ámbito de la escuela. Ya que el uso del contraejemplo como herramienta mediadora, favoreció, en el debate, el establecer relaciones entre diferentes definiciones de un mismo objeto o entre definiciones similares (como la continuidad, continuidad uniforme, continuidad absoluta, entre otras).

\section{CONCLUSIONES}

La puesta en desarrollo de un debate científico para la construcción del concepto de función convexa, resultó ser un escenario propicio, en el cual, el uso del contraejemplo jugó un papel revelador, como una herramienta didáctica mediadora entre la formulación de conjeturas, y refutación y/o afinación de formulaciones acerca del concepto. En este proceso emergieron nociones fundamentales en torno al de función convexa, mismas, que tras su discusión se logró preparar el terreno para hacerlas evolucionar teóricamente hacia las determinadas definiciones como se muestra en las definciones de 2 a 7 de la Tabla 2.

La preparación por parte del professor en los aspectos: epistemológico, didáctico y cognitivo del concepto matemático: Función convexa, favoreció el papel que jugó en su intervención en el debate, ya que en todo momento se tuvo el cuidado de no desviar las discusiones hacia situaciones ajenas y alejadas del contenido matemático en cuestión. Esta preparación permitió en los estudiantes proponer conjeturas y debatir sobre ellas, en dichos debates se utilizo el contraejemplo como argumento para aceptar o refutar las propuestas de definiciones. De esta manera, se posibilitó a travéz de la definición 2, Tabla 2, la evolución de las definiciones.

Con el presente trabajo se contribuye en una propuesta didáctica para el tratamiento de los conceptos y sus definiciones en un ambiente de debate científico, en el que la situación del contraejemplo ofrece una herramienta de corte didáctico que posibilita la formulación y refutación de conjeturas, favoreciendo la evolución de las definiciones generadas por la prueba, y tomando como punto de partida para análisis de las definiciones de tipo cero.

\section{AGRADECIMIENTOS}

Este trabajo fue financiado, en parte, por el Proyecto FOMIX-CONACyT-UAGro 249818.

\section{REFERENCIAS}

Bartle, R. y D. Sherbert, Introducción al Análisis Matemático de una Variable, 3aㅡ Ed., Limusa - Wiley, México (2005)

Brousseau, G., Iniciación al Estudio de la Teoría de las Situaciones Didácticas, Libros Zorzal, Bs. Aires, Argentina (2007) 
Brousseau, G., Educación y Didáctica de las Matemáticas, en Educación Matemática, México (1999)

Brousseau, G., Los Diferentes Roles del Maestro en Didáctica de Matemáticas, Aportes y reflexiones, en C. Parra e I. Saiz, Eds., Paidós Educador, Ecuador (1994)

Brousseau, G., Fundamentos y Métodos de la Didáctica de la Matemática, Serie B, Trabajos de Matemática, № 19 (1986)

Bustos, A. S. y G. Zubieta, Descubrimiento de Conocimiento Matemático Mediante la Reformulación de Conjeturas Falsas en un Ambiente de Pruebas y Refutaciones, Enseñanza de las Ciencias, 33(3), 117-136 (2015)

Calvo, C., Un Estudio Sobre el Papel de las Definiciones y las Demostraciones en Cursos Preuniversitarios de Cálculo Diferencial e Integral, Tesis Doctoral Publicada, Bellaterra, Barcelona (2001)

Díaz-Barriga, A., Didáctica y Currículum, Articulaciones en los Programas de Estudios, Paidós, México (1996)

García, O. y L. Morales, El Contraejemplo como Recurso Didáctico en la Enseñanza del Cálculo, Revista Iberoamericana de Educación Matemática, 13, 161-175 (2013)

Giannakoulias, E., E Mastorides, D. Potari y T. Zachariades, Studing Teachers' Mathematical Argumentation in the Contexto of Refuting Students Invalid Claims, The Journal of Mathematical Behavior, 29, 160-168 (2010)

Granville, W., Cálculo Diferencial e Integral, 1aㅡ. Ed., Limusa, México (2009)

Huang, Ch., Engineering Students' Generating Counterexample of Calculus Concepts. Global Journal of Engineering Education, 16(2), 93-97 (2014)

Kleene, S., Logique Mathématique, North-Holland Publishing Company, Amsterdam (1967)

Klymchuk, S., Counterexamples in Cálculus.Mathematical Association of América, Resource Materials, United States of América (2010)

Knuth, E.J. e Y. Ko, Validating Proofs and Counterexamples Across Contente Domains: Practices of Importance for Mathematics Majors, The Journal of Mathematical Behavior, 32, 20-35 (2013)

Komatsu, K., Counter-examples for Refinement of Conjectures and Proofs in Primary School Mathematics, The Journal of Mathematical Behavior, 29(1), 1-10 (2010)

Komatsu, K., K. Jones, T. Ikeda y A. Narazaki, Proof Validation and Modification in Secondary School Geometry, The Journal of Mathematical Behavior, 47, 1-15 (2017)

Lakatos, I., PreuvesetRefutaions: Essai Sur la Logique de la Decouverte Mathématique, Herman, Paris (1984)

Legrand, M., Débat Scientifique en Cours de Mathématiques et Specifité de L'analyse, Repères IREM, 10, 123-159 (1993)

Martínez, R. y P. Detzel, El Lugar que Ocupa el Contraejemplo en la Enseñanza de la Matemática, III Reunión Pampeana de Educación Matemática, Volumen III, Santa Rosa, La Pampa, Argentina (2010)

Morales, A., C. Dolores y otros tres autores, Methodology Based on Problem Solving in the Treatment of the Concept of Limit to Infinity, Inter. Journal Researchin Education Methodology, 5(1), 542-550 (2014)

Piskunov, N., Differential and Integral Calculus, 3를. Eol. I (1), MIR, Moscow (2004)

Pisot, C. y M. Zamansky, Matemáticas Generales. Algebra y Análisis, Ed. Montaner y Simón, Barcelona, España (1972)

Polya, G., Les Mathématiques el le Raisonnement Plausible, Gauthier-Villar, París (1958)

Polya, G., How to Solve It? 2ª Ed., Doubleday \& Co., New York, USA (1957)

Ramos, G. y A., López, La Formación de Conceptos: Una Comparación Cognitivista y Histórico-Cultural, Educação e Pesquisa, 41(3), 615-628 (2015)

Robinet, J., Le Pourquoi et le Commentd ‘unelngenierie (La Convergence Uniforme), Publicaciones IREM 12, (1992)

Rudin, W., Functional Analysis, McGaraw Hill, New York (1973)

Rudin, W., Principios de Análisis Matemático, 3ª Ed., McGraw Hill, Madrid (1980)

Rudin, W., Análisis Real y Complejo, 3aㅡ. Ed., McGraw Hill, Madrid, España (1988)

Spivak, M., Calculus, $3^{a}$ Ed., Cambridge Univ Press, USA (2003)

Weber, K., How Syntactic Reasoners can Develop Undertstanding, Evaluate Conjetures, and Generate Counterexamples in Advanced Mathematics, The Journal of MathematicalBehavior, 28, 200-208 (2009)

Winicki, G., Las Definiciones en Matemáticas y los Procesos de su Formulación: Algunas Reflexiones, Acta Latinoamericana de Matemática Educativa, 19, 528-537(2006)

Zazkis, R. y E. Chernoff, What Makesa Counterexample Exemplary? Educational Studies in Mathematics, 68,195-208 (2008)

Zilmer, W., Metodología de la Enseñanza de la Matemática I, Tres partes, Ed. Pueblo y Educación, Ciudad de la Habana (1981) 